\title{
LA CONCEPCIÓN DE CLIENTE Y SU REPERCUSIÓN EN LA CALIDAD DEL SERVICIO EN EL RESTAURANTE.
}

\author{
Aureliano del Toro Cabrera, MsC. \\ Máster en Gestión Turística (Cuba). \\ Docente en la Carrera de Administración de Empresa en el \\ Instituto Superior Tecnológico Bolivariano, Ecuador. \\ adeltoro@itb.edu.ec
}

\section{ARTÍCULO DE REFLEXIÓN}

Recibido: 17 de mayo de 2019.

Aceptado: 30 de agosto de 2019.

\section{RESUMEN}

Con la intención de describir la percepción que existe en los restaurantes sobre la calidad del servicio se intercambió criterios con estudiantes, amistades y colegas para saber sus opiniones sobre cómo fueron atendidos durante su estancia en los restaurantes objetos de estudio. Mediante un paradigma interpretativo se analizó la recolección de los datos en diferentes grupos focales teniendo en cuenta las experiencias expuestas por los participantes, que fueron plasmadas mediante un cuaderno de notas. La diversificación de términos como usuario, comensal o consumidor que existen para definir a la persona que acude al restaurante crea confusión, esto produce consecuencias negativas en el personal de servicio, siendo necesario un cambio de percepción donde las personas tienen que reaprender sobre la nueva concepción de cliente basadas en las relaciones interpersonales.

Palabras clave: concepción, cliente, restaurante, servicio.

\section{ABSTRACT}

With the intention of describing the perception that exists in restaurants, it's exchanged criteria with students, friends and colleagues to know their opinions about how they were served during their stay in the restaurant. Through an interpretative's paradigm, the data collection in different focal groups was analyzed taking into account the experiences presented by the participants which were expressed through a notebook. The diversification of term as a user, diner or consumer that exists to define the person who goes to the 
restaurant creates a disorder that produces consequences with the restaurant's staff, being necessary a change of perception, where people have to relearn about the new client conception, Based on interpersonal relationships

Keywords: conception, customer, restaurant, service.

\section{INTRODUCCIÓN}

En la actualidad las empresas que quieren triunfar en el sector de los servicios, tienen que abandonar la estrategia competitiva estructurada en los productos para establecer una nueva política basada en la excelencia y calidad de los servicios, capaz de superar las expectativas de los clientes, en la que la actitud que asuma el personal de servicio durante su desempeño laboral incida directamente. "Calidad significa brindar lo que se le prometió desde el diseño del producto o servicio. La excelencia significa ir más allá de las expectativas del cliente, sorprenderle de manera positiva" (Pérez, 2010, p. 23).

Definir con claridad cuáles son los atributos del servicio que inciden en el cliente para lograr la excelencia debería ser uno de los principales requerimientos de las empresas que se relacionan con personas en cualquier lugar del mundo. Por eso, se considera que "constituye un imperativo para el sector de los servicios, comprender la importancia que representa gestionar afectivamente las relaciones interpersonales para lograr matices bien definidos que marquen la diferencia entre la competencia y la excelencia” (Del Toro, 2015, p. 83).

Con la intención de describir la percepción que existe en los restaurantes de la zona norte de la ciudad de Guayaquil sobre la atención poco personalizada hacia los clientes, cuando estos acuden al restaurante, se intercambió criterios con estudiantes, amistades y colegas para saber sus opiniones sobre cómo fueron atendidos durante su estancia en dichos restaurantes. Considerando que el personal de servicio podría realizar técnicamente bien su trabajo en el salón, pero si no interactúan de manera afectiva con las personas que atienden, la situación se torna automatizada o robótica.

De manera generalizada se pudo percibir que, durante el servicio en los restaurantes, no se presta la atención que requiere incentivar las relaciones interpersonales durante los llamados momentos de la verdad, donde el personal de servicio tiene la oportunidad de 
demostrar su profesionalidad al exteriorizar un conjunto de normas y rasgos actitudinales, que complementa el conocimiento técnico que estos tienen. Compartiendo esta misma idea se dice que "Las competencias actitudinales se relacionan directamente con el "saber-ser" o "saber-actuar" frente a una situación determinada. Por esta razón son competencias extremadamente útiles para nuestro desempeño profesional y sin las cuales muchos de nuestros conocimientos no podrían ser aprovechados” (Valdez, 2016, ๆ1).

En aras de establecer una interpretación crítica sobre la concepción que se tiene sobre el cliente, con el objetivo de evitar implicaciones inadecuadas en la actividad del servicio de alimentos y bebidas en el sector de la restauración, se considera que es improcedente el uso de determinados términos como usuario, consumidor o comensal para definir a las personas que acuden a un restaurante para disfrutar de un atractivo momento en ese lugar. Una concepción errónea sobre el empleo de un término puede implicar una acción involuntaria (léase inapropiado), de las personas que realizan el servicio.

Este comportamiento no está regido por leyes generales, por eso la interpretación poco clara de un término pudiera estar caracterizada por regularidades culturales subyacentes que determinen el trato hacia el cliente. "Si en un contexto experimental, proporcionamos a los sujetos de la investigación un conjunto de estímulos generados teniendo presente una serie de reglas simples, inconscientemente los sujetos experimentales inferirán las regularidades subyacentes" (Redolar, 2012, p. 109). Estas regularidades formativas en el personal de servicio al escoger ciertas terminologías inadecuadas para definir a la persona que acude al restaurante, pueden distorsionar o subyacer la verdadera concepción de cliente.

El término concepción es utilizado como la acción y el efecto de concebir, donde en unas de sus acepciones lo considera como comprender algo, encontrarle justificación o formarse una idea o un designio de algo en la mente de las personas. Partiendo de este criterio se hará un análisis para conocer el estado del arte que existe con respecto a la concepción de clientes según las diferentes fuentes literaria. En investigaciones previas sobre el tema se hace referencia a la atención al cliente, comenzando por la tradicional escala SERVQUAL (Parasuraman, Zeithaml y Berry, 1993), cuyos investigadores definen la calidad de servicio a partir de cinco dimensiones, donde cuatro de ellas hacen referencia a los aspectos intangibles del servicio, mientras que solamente una a los aspectos tangibles. 
En esta época Grönroos (1994) establece un modelo que refleja la percepción del servicio a partir de una serie de interacciones que se producen entre el cliente y el personal de la empresa, que van a repercutir en la manera en que los clientes perciben la calidad de los servicios que están recibiendo. "Básicamente, la calidad de un servicio, tal y como es percibida por los clientes tiene dos dimensiones, a saber, una dimensión técnica o de resultado y una dimensión funcional o relacionada con el proceso" (p. 37). Por primera vez se establece que la calidad del servicio no solo se puede concebir a partir de las especificaciones técnicas del producto (calidad técnica) como se consideraba hasta este momento, sino que, además también influye en la percepción de la calidad un componente funcional relacionado con la manera en que se le transfiere ese bien al cliente. Aun no se hace énfasis en la importancia que requiere, considerar las competencias actitudinales del personal de servicio durante las relaciones interpersonales.

La concepción que tiene la literatura técnica sobre el término de cliente, es considerada como muy escueta e imprecisa. Analizando las definiciones que exponen la Organización Internacional de Normalización (ISO), entidad encargada de garantizar la calidad (ISO 9000:2005), desde un inicio ha concebido los términos de consumidor y usuario en su definición, además solo toma en cuenta la parte tangible del proceso para definir al cliente (cliente: organización o persona que recibe un producto), es decir, la calidad técnica, obviando algo tan importante como es la intangibilidad del servicio. No ha sido hasta la actualidad (ISO 9000:2015) que incorporan la actividad del servicio a la normativa para definir al cliente (cliente: persona u organización que podría recibir o que recibe un producto o un servicio destinado a esa persona u organización o requerido por ella), que como ya se ha expresado se considera demasiado escueta e imprecisa.

Finalmente, analizando el acervo cultural que existe en la actualidad sobre las personas que acuden al restaurante es preciso aclarar que términos como usuario, comensales y consumidor son incoherentes para definir la verdadera concepción a tener en cuenta sobre el cliente. Para comprender mejor dicha afirmación es necesario interpretar la definición que exponen fuentes como la RAE, Diccionario Manual de la Lengua Española Vox. Larousse Editorial, S.L. Diccionario de la lengua española Espasa-Calpe y otras fuentes sobre estos términos, para corroborar que no son coherentes para definir a la persona que acude al restaurante. Por lo que es necesario precisar con urgencia esta situación, debido a que el alto dominio que existe en el acervo cultural de estos términos 
puede repercutir de manera involuntaria en las literaturas científicas y técnicas, afectando el desempeño laboral del personal de servicio en el sector de los servicios.

En el sector de la restauración, esta investigación pretende describir de forma detallada las repercusiones que pueden afectar en la actividad del servicio, a partir de la concepción que asuma el personal de servicio sobre la persona que acude al restaurante para disfrutar de esos momentos, al deleitarse de una deliciosa comida. Una interpretación imprecisa sobre la concepción de la persona que acude a un restaurante a solicitar los servicios de alimentos y bebidas, puede generar implicaciones inapropiadas (involuntaria) durante la generación del servicio. Según este planteamiento surge la siguiente interrogante: ¿cómo es la concepción de cliente y sus repercusiones en la calidad del servicio en el restaurante?

\section{MATERIALES Y MÉTODOS}

La investigación cualitativa reside en la particularidad de estudiar el comportamiento de los fenómenos sociales, a partir de una metodología que respete su naturaleza. El carácter subjetivo y complejo de estos procesos requiere de un análisis sistémico que facilite conocer las percepciones de los sujetos que son objeto de estudio, para diferenciar entre lo propuesto por el investigador y lo que aporta las personas que se investigan, prestando especial atención a la información de carácter analítico que se obtiene de dicha investigación. "Esto significa que los investigadores cualitativos estudian las cosas en su situación natural, tratando de entender o interpretar los fenómenos en términos de los significados que la gente les otorga." (Denzin y Lincoln, 2012, p.2). Los procedimientos durante esta investigación se establecieron a partir de grupos previamente establecidos que describieron su percepción sobre la problemática que ha sido objeto de estudio.

Bajo la perspectiva de un paradigma interpretativo enfocado en analizar problemas de tipo social para darle solución, se ha querido describir la percepción que se tiene sobre las personas que acuden al restaurante, para luego de un proceso de comprensión, poder clarificar las acepciones que tienen en cuenta para definir a estas personas. Según Cano (2009), "el paradigma interpretativo se centra en el mundo de los significado y valores desarrollados dentro de la organización y que conforman su cultura, acentuando el carácter ambiguo, incierto y complejo de la misma" (p. 197). Esto demuestra la urgente necesidad de incidir de forma consciente en el acervo cultural que coexisten sobre estos términos en 
los locales del sector de la restauración, donde de forma habitual se le considera como normal el uso de cualquiera de ellos para definir al cliente. Esta deficiencia en el conocimiento de los prestadores del servicio es necesario aclararla, estandarizándolas para que todos puedan entender de una manera más específica su significado.

Esta investigación tuvo un enfoque fenomenológico, al considerar que el mundo es aquello que se percibe a través de la conciencia del individuo, y que es interpretado según sus experiencias. A través de este enfoque se realizó una "descripción concreta de una experiencia dada por una persona específica quien es vista no como objeto distante de investigación, sino más bien como co-investigador porque participa creando su propia experiencia a la interpretación de dicha experiencia" (De Castro, 2011, p. 21). El estudio de la realidad objetiva que se manifiesta en el restaurante, expuestas a través de los criterios y experiencia de las personas que conformaron la investigación, facilitó interpretar el significado de la actividad de los servicios, bajo una perspectiva ética, teniendo en cuenta un conjunto de actitudes y virtudes internas que el personal de servicio debería exteriorizar, en función de las percepciones adquiridas.

Con la finalidad de lograr establecer una situación lo más real posible, este estudio se centró en la técnica de grupos focales, al definir como muestra para nuestro estudio a un grupo de estudiantes, amistades y colegas de forma intencional, por considerarlos como informantes claves, debido a que ellos han vivido de una forma u otra la experiencia dentro del salón cuando han estado en el rol de cliente, de ahí que se consideren que tienen toda la autoridad para exponer su percepción sobre el servicio recibido. "Un diseño de investigación sitúa a los investigadores en el mundo empírico y los relaciona a los lugares específicos, a las personas, grupos, instituciones y cuerpos de materiales interpretativos relevantes, lo que incluye documentación y archivos" (Denzin y Lincoln, 2012, p. 24).

Durante las secciones de trabajo se formularon diferentes preguntas semiestructuradas que permitieron a los informantes exponer sus criterios e interactuar entre ellos sobre la concepción que tienen de los términos analizados, con el fin de llegar a un consenso en común, que dieran credibilidad y validez a los datos procesados. Esta técnica de participación, donde se expusieron las vivencias experimentadas en cada uno de los restaurantes, fue de mucha utilidad para la investigación, ya que ayudó a consolidar los conocimientos teórico-práctico, aportado información durante las sesiones realizadas con 
los grupos focales, a partir de las experiencias y percepciones adquiridas por los participantes durante el servicio en los restaurantes.

El cuaderno de notas es el instrumento utilizado en esta investigación para registrar todos los criterios y opiniones expuestas por los participantes durante las sesiones de trabajo, para luego ser interpretadas, por eso "constituye una fuente importante para ponderar la información" (Toro, 2006, p. 179). En este sentido, se convierte en una herramienta importante que permitió sintetizar las experiencias de los informantes que después sirvieron para analizar los resultados, además ayudó a organizar los datos (información) obtenidos durante los grupos focales, constituyó "un recurso clave que permite al investigador tener dominio del proceso de indagación" (Toro, 2006, p. 339).

La dialéctica del discurso se establece a partir de la discusión de los diferentes criterios fundamentados entre los tres actores involucrados: por un lado, tenemos el apoyo bibliográfico establecido por la comunidad científica, por el otro, los resultados relevantes de los participantes y finalmente la opinión personal del investigador, que según RebolledoMalpica (2019) "el objetivo de esta triangulación de técnicas de recolecciones de datos fue llegar a la saturación de los mismos" (p. 92). El análisis de los datos a partir de la triangulación de la información adquirida por los tres actores involucrados, garantizan el rigor científico de esta investigación.

\section{DISCUSIÓN Y RESULTADOS}

Gestionar satisfactoriamente las relaciones interpersonales resulta fundamental para el desarrollo integral de la persona, al obtener una gratificación de la sociedad. A través de ellas el personal de servicio obtiene un importante refuerzo desde el punto de vista social del entorno inmediato en que se desenvuelve, que favorece su desempeño laboral de la actividad que realiza con sus clientes. La persona que proporciona un servicio es el responsable directo e inmediato en producir la lealtad del cliente, donde se precisa que actúe como mediador en la creación de las experiencias de servicios en el cliente. De acuerdo con este criterio, la educación emocional facilita la formación de actitudes positivas ante la vida, que favorece el desarrollo de habilidades actitudinales que ayudan en el desempeño laboral del personal de servicio. 
Basándose en la realidad objetiva expresada por el autor, el resultado del estudio cobra relevancia, cuando uno de los participantes del grupo focal afirmó «a estas personas no les interesa el cliente, para ellos solos somos simples consumidores». Querer otorgarle categorías como usuario, consumidor o comensal, sería un poco intrascendente, por eso este trabajo pretende realizar un análisis exhaustivo sobre estos términos con la finalidad de exponer una nueva concepción que permita cambiar la filosofía de servicio que existe en la actualidad hacia el cliente.

La psique actúa como el conjunto de capacidades humanas que abarca los procesos conscientes e inconscientes de estas personas, a través de las percepciones, sensaciones, emociones y sentimientos, que adoptan una posición activa frente al reflejo de la realidad de cada uno de los estímulos del medio en que se desarrolla, "permitiéndole la supervivencia en función del desarrollo que le permita su inteligencia. Al adquirir un carácter eminentemente consciente, le permite no sólo adaptarse al medio sino también transformarlo para su provecho" (Bonilla, 2014).

Desde la psicología se concibe que el cerebro realiza la actividad psíquica en los seres humanos, por lo que se convierte así en la designación de todos los procesos y fenómenos que hacen la mente humana como una unidad (Barrios, 2012). La percepción influye considerablemente en el conocimiento que se tenga sobre cualquier aspecto de la vida social, de ahí que el personal de servicio pueda considerar que su función principal es la de ofertar el producto que va a ser consumido o usado por la persona que atiende, obviando su principal rol que es la de relacionarse con él. Por eso la percepción que estos sean capaces de representarse sobre la concepción de cliente, podría degradar inconscientemente el servicio al tratar a la persona que atiende como consumidor o usuario del restaurante, en vez de ser considerado como el ente principal del local. Esto se refleja en los resultados del estudio al opinar que «yo me sentí como una mercancía, y no como una persona que viene a pasar un rato agradable».

Esta investigación demuestra que el éxito durante el servicio, no depende tanto del nivel de inteligencia lógico-matemática que el personal tenga, sino más bien de las habilidades que posea para manejar contextos interpersonales. Corroborado por lo que dicen los informantes «yo vengo a este restaurante, porque me tratan como familia». Según Bisquerra (2003) “la educación emocional es un proceso educativo, continuo y permanente, que pretende potenciar el desarrollo de las competencias emocionales como elemento 
esencial del desarrollo integral de la persona, con objeto de capacitarle para la vida" (p. 27). De aquí se deduce la importancia de considerar al cliente en el restaurante como la figura principal, el rey que merece todo los honores y reconocimientos posibles.

Oliveira (2007) fundamenta su investigación sobre la importancia que representa la culinaria como atracción principal que motiva el desplazamiento de personas, al considerar que "el acto de comer fuera de casa tiene una función social realmente importante ya que, además de permitir estrechar relaciones entre los que viajan en grupo, puede crear nuevas relaciones e interacciones con otras personas" (p. 263). Según los participantes la comida típica que ofrece determinada región no debería constituir el atributo principal que garantice la calidad del servicio, cuando han expresado «la comida no es lo único importante de este restaurante, también me complace el trato que he recibido»; argumentación que es válida, si se considera que también se debería tener en cuenta la gran importancia que representan las relaciones interpersonales durante estos encuentros de servicios para lograr la excelencia.

Se está consciente que la calidad técnica, que representa los aspectos tangibles, incide en la percepción final que tienen los clientes sobre el servicio, pero de una manera determinante también influyen los aspectos intangibles del servicio. Podría afirmarse que el cliente percibe y valora más los aspectos intangibles del servicio que los propios tangibles, razón más que importante para incidir sobre ellos.

En el Primer Simposio Brasileño de Ciencia de los Servicio José Roberto Concha, plantea que se decidió realizar nuestra investigación, tomando en cuenta las siguientes variables para medir la satisfacción del cliente en los restaurantes: calidad de servicio, calidad de producto y precio (Concha, 2010). Sin embargo, los participantes del estudio consideran que la satisfacción del cliente no está solamente en función del producto o rapidez con que se les sirve en la mesa, sino más bien en la manera en que se gestionen las relaciones interpersonales. "No nos interesa el precio a pagar por la comida, sino la manera en que seamos tratados durante la estancia en el restaurante». Se quiere dejar por sentado que para garantizar la fidelidad de los clientes es necesario superar sus expectativas, es decir, ofrecerle durante la realización del servicio mucho más de lo que ellos esperan recibir.

Gomes, De Azevedo y Gomes (2013) argumentan que "el sistema de oferta de los restaurantes de alta gastronomía, consideran que no se limita únicamente a las 
preparaciones culinarias, sino que también abarca el lugar, su arquitectura, la decoración y la escenificación de los servicios" (p. 338). Los participantes reconocen que aspectos como el olor, la temperatura, la música, entre otros, pueden revelarse como elementos capaces de crear placer a los clientes; pero que este deseo no sería capaz de lograse sino se complementa con un buen trato y amabilidad. Reafirmamos el criterio de los participantes al considerar que las relaciones interpersonales constituyen la piedra angular donde se sustenta todas las empresas que quieran lograr la excelencia.

Todas estas imprecisiones expuestas sobre la actividad de los servicios en el restaurante del entorno norte de Guayaquil está motivada por dos causas fundamentales, la primera es por el uso improcedente de determinados términos para definir a una persona, donde desde el punto de vista de la programación neurolingüística, se aprecia que la utilización de diferentes términos para designar a la persona que acuden a solicitar los servicios de restauración son incoherentes con la concepción de cliente, en segundo lugar se quiere manifestar la manera en que puede influir en la psiquis humana el significado de cliente si se parte del hecho de que la interpretación que las fuentes exponen del término es muy escueta y poco expresiva.

No se pretende restarle importancia a la calidad técnica de un producto, mucho menos a las especificaciones técnicas que demandan los clientes, lo que se quiere hacer entender es que se comprenda la gran influencia que tiene en la percepción de la calidad del servicio fortalecer los vínculos que se establecen durante esta actividad para lograr el éxito. En la actualidad se debe pretende ir más allá de la certificación, al establecer una cultura de servicio, enfocada a superar las expectativas de los clientes, fundamentada en intensificar las relaciones interpersonales que se establecen con los clientes durante los diferentes momentos de la verdad para lograr fidelizarlo y alcanzar el éxito deseado.

Según el imaginario colectivo se interpreta el término usuario como las personas que tienen derecho a hacer uso de un servicio o adquirir un producto, sin especificar la manera en que deberían realizarse estas transacciones. Otras fuentes lo relacionan con la persona que utiliza una computadora, sistema operativo o cualquier sistema informático, además del uso de otros servicios mecanizados como los cajeros automáticos. Los participantes concuerdan que «hemos sido llamados como usuario en estos lugares, además de escucharlo repetitivamente en diferentes medios». Como se puede apreciar esta definición, aunque es correcta para la conceptualización que se quiere expresar del término, no 
expresa categóricamente la concepción tan importante que requiere otorgarle a la persona que solicita el servicio de alimentos y bebida. Recuérdese que la razón de ser de un restaurante es el cliente, una empresa existe porque existen ellos, por eso minimizar la consideración hacia ellos sería un fracaso total.

La Real Academia Española (RAE), el diccionario de la lengua española Espasa-Calpe y otras bibliografías interpretan el concepto de consumidor como de un uso muy extendido en el idioma español y especialmente es empleado a instancias del ámbito de la economía para denominar a aquel individuo u organización que demanda bienes o servicios que ofrece, ya sea un productor o quien provee los mencionados bienes y servicios. Durante las discusiones del grupo focal los informantes coinciden en que «hemos sido llamados como consumidores en estos lugares, causándonos una mala impresión». Esta definición, aunque un poco más aceptada que la anterior, considera de una manera muy general a la persona que compra productos o contrata servicios en el sector de la restauración, como el consumidor final dentro del proceso productivo, conllevando al gravísimo error de no considerar los aspectos intangibles como las relaciones interpersonales durante este proceso.

Larousse Editorial, S.L. y otras fuentes, interpretan el término comensal como cada una de las personas congregadas y reunidas que comen en la misma mesa ya sea de una sola familia o de algunos invitados. Este término es utilizado en el sector de la restauración, uso que se evidencia en las opiniones de los participantes de esta investigación cuando expresan que «en estos lugares nos nombran como comensales, sin tomarnos en cuenta». Este último término da la impresión que es aquella persona que se sienta en una mesa, consume los alimentos y se retira, obviando de igual forma las relaciones interpersonales que se establecen durante la entrega de los alimentos y bebidas en el restaurante.

Como se aprecia las vivencias en el ser humano forman parte de su espiritualidad, de modo que este tipo de reflejo crea una unidad indisoluble entre lo objetivo y lo subjetivo. Conceptualizar el significado de un término es algo real, objetivo, que se manifiesta en diferentes actos externos y en la conducta de las personas, razón por la cual el interpretar erróneamente un término conlleva a actuar de una manera inapropiada cuando se relacionan con los clientes. Las empresas de servicios deben fomentar una estrategia capaz de volver a su personal verdaderos fanáticos de las actividades que realizan con la finalidad de lograr atraer, mantener y desarrollar relaciones afectivas con sus clientes. Es decir, 
convertirse en verdaderos amantes del servicio, ya que "la fidelización del cliente es una tarea de vital importancia para la supervivencia de la empresa" (Bastos, 2007, p. 14).

La competencia en el sector de los servicios exige cambios sustanciales sobre los conceptos de productos y servicios para poder ser más competitivos. Establecer una estrategia de servicio encaminada a transformar los componentes tangibles implícitos durante las relaciones interpersonales con los clientes, constituye la piedra angular donde se debe sustentar el servicio para lograr el éxito en cualquier empresa. De esta manera se evidencia la importancia que representa en el sector de los servicios, establecer competencias actitudinales (Saber Ser) durante el desempeño laboral en todas aquellas personas que trabajan de cara al cliente, por encima de las especificaciones técnicas del producto. Se ha comprobado que los clientes prestan mayor interés a las funciones afectivas establecidas durante los encuentros de servicios que a cualquier otro atributo presente en el mismo.

Considerando un enfoque hacia el cliente bajo la premisa de las relaciones interpersonales, se pretende establecer una connotación diferente del término cliente donde se consideren una serie de atributos que incidan significativamente en la percepción del servicio que están recibiendo. Este trabajo pretende dejar por sentado que el concepto de cliente se debe fundamentar bajo la premisa de las relaciones interpersonales como punto de partida para lograr el éxito. Considerando además al servicio como "una serie de actividades o procedimientos, de naturaleza esencialmente intangible, enfocados a gestionar relaciones interpersonales afectivas que garanticen en el cliente un conjunto de experiencias, sensaciones y sentimientos capaces de superar las expectativas creadas, con la finalidad de fidelizarlos" (Del Toro, 2016, p. 34).

\section{CONCLUSIONES}

La finalidad de esta investigación fue describir la percepción de la realidad objetiva que existe sobre la concepción de clientes desde la perspectiva de los diferentes actores para luego transformarla en la contextualización de un nuevo conocimiento. La diversificación de término para definir a la persona que acude al restaurante crea un desorden que produce diferentes consecuencias en el personal de servicio, que puede llegar hasta menospreciar a estas personas por no tener una definición clara del término. Enfatizar en las relaciones interpersonales como la piedra angular para lograr el éxito de cualquier empresa, 
considerando una nueva concepción del término de cliente es el objetivo principal de este trabajo.

Los resultados en el análisis de la concepción de cliente y sus repercusiones en la calidad del servicio en el restaurante en el sector norte de Guayaquil, evidenció que el establecimiento de relaciones interpersonales afectivas en la atención al cliente no constituye una prioridad en la oferta del servicio, al observar que los sujetos estudiados no se sienten totalmente complacidos con el servicio recibido como clientes. Aspecto que está alejado de lo que proponen los expertos en el tema.

Como reflexión de esta investigación se considera concluyente que la comprensión sobre la actividad de los servicios es eminente, donde las personas tienen que reaprender una nueva concepción de cliente considerándola como la persona natural o jurídica que tiene una serie de necesidades e inquietudes que son oportunamente satisfechas cuando acuden a una empresa para hacer uso de sus productos y/o servicio, mediante relaciones interpersonales afectivas capaces de crear experiencias de servicios que superen las expectativas. Se considera la razón de ser de una empresa, pues son ellos los que, mediante una transacción financiera u otro medio de pago, garantizan el éxito y su prosperidad.

Esta definición más exhaustiva y tal vez completa, es debido a que se pretende que las personas que laboran en el sector de los servicios comprendan la necesidad de dejar el uso de los términos de usuario, consumidor y comensal, para identificar al cliente como la persona más importante que acude al establecimiento. Siendo necesario articular las actuaciones de ambos dentro de un marco real y afectivo, donde el servicio gire en torno a una serie de actividades o procedimientos previamente diseñados en el que las relaciones interpersonales sean el factor principal.

\section{REFERENCIAS BIBLIOGRÁFICAS}

Alcaide, J. (2015). Fidelización de clientes. México: Editorial ESIC.

Barrios, Y. (26, noviembre, 2012). La psiquis humana. [Mensaje en un blog]. Recuperado de http://elrinconuniversitariodetareas.blogspot.com/2012/11/la-psiquishumana.html.

Bastos, A. (2007). Fidelización Del Cliente. Pontevedra, España: Ideas propias Editorial S.L. 13 
Bisquerra, R. (2003). Educación emocional y competencias básicas para la vida. Revista de Investigación Educativa, 21(1), 7-43.

Bonilla, L. (2014). El paradigma paradójico: O la historia del ser. Bloomington: Palibrio.

Cano, E. (2009). Estrategias e instrumentos para la autoevaluación. Barcelona, España: Ministerio de Educación.

Cabrera, S. (Ed.) (2013). La fidelización del cliente en negocios de restauración. Cuadernos del Centro de Estudios en Diseño y Comunicación, 45(1), 155-165.

Cadena, J. M.; Vega, A.; Real, I. y Vásquez, J. (2016). Medición de la calidad del servicio proporcionado a clientes por Restaurantes en Sonora, México. Ingeniería Industrial. Actualidad y Nuevas Tendencias, 5(17), 41-60.

Cevallos, R. P.; Carreno, D. V.; Peña, I. V. y Pinargote, K. G. (2018). Evaluación de la calidad del servicio: Un paso más cerca a la objetividad. San Gregorio, 4(18), 49-59.

Concha, V. J. (noviembre, 2010). Satisfacción del Cliente en Empresas de Servicio: El Caso de la Industria de Restaurantes. Presentado en el Primer Simposio Brasileño de Ciencias de los Servicios, Universidad Icesi, Brasilia, Brasil.

Croxatto, L. (2005). Creando valor en la relación con sus clientes. Buenos Aire, Argentina: Dunken.

De Castro, A. (2011). Psicología Clínica. Fundamentos existenciales. Bogotá, Colombia: Universidad del Norte.

Del Toro, A. (2015). Gestión de las relaciones interpersonales para lograr el éxito en las empresas de servicios. Revista Interamericana de Ambiente y Turismo, 11(1), 75-83.

Del Toro, A. (2016). Técnicas del Servicio en el Restaurante. Guayaquil, Ecuador: EDITOGRAN.

Denzin, N., y Lincoln, Y. (2012). Manual de investigación cualitativa. Paradigmas y perspectivas en disputa. (vol. II). Barcelona: Gedisa.

García, J. y Martínez, M. I. (2019). Uso de la dotación básica del Restaurante y asistencia en el Pre servicio. Antequera, España: IC Editorial.

Gomes, A., de Azevedo, M., y Gomes, A. (2013). El sistema de oferta de restaurantes de alta gastronomía. Una perspectiva sensorial de las experiencias de consumo. Estudios y perspectivas en turismo, 22(.2), 336-356.

Grönroos, C., y Kotler, P. (1994). Marketing y gestión de servicios. Madrid, España: Ediciones Díaz de Santos.

Gwo, T. \& Hung, C. (2011). Applying Importance-Performance Analysis as a Service Quality Measure in FoodService Industry. Journal of Technology Management \& Innovation, $6(3), 106-114$. 
Hernández, D., Fernández, D., y Baptista, L. (2010). Metodología y Técnicas de la Investigación. México: Interamericana Editores, SA DE C.V.

Heskett, J., Earl, W., y Hart, C. (1993). Cambios creativos en servicios. Madrid, España: Díaz de Santos.

ISO 9000. (2005). Sistemas de gestión de la calidad - Fundamentos y vocabulario. Recuperado de https://www.iso.org/obp/ui/\#iso:std:iso:9000:ed-3:v1:es:term:3.2.5

ISO 9000. (2015). Sistemas de gestión de la calidad - Fundamentos y vocabulario (4 ${ }^{\text {ta }}$ edición). Recuperado de http://fing.uncu.edu.ar/catedras/cialimentaria/unidadestematicas/ISO\%209000-2015.pdf

León, M. y Díaz, E. (2019). Recursos humanos y dirección de equipos en restauración (2ª edición). Madrid, España: Ediciones Paraninfo, S.A.

Oliveira, S. (2007). La importancia de la gastronomía en el turismo. Un ejemplo de Mealhada - Portugal. Estudios y perspectivas en turismo, 16(3), 261-280.

Parasuraman, A., Zeithalm, V., \& Berry, L. (1993). SERVQUAL: A multiple-item scale for measuring consumer perceptions of service quality. Journal of Retailing, 64(1), 12-40.

Pérez, V. (2010). Calidad Total en la Atención Al Cliente. España: Ideaspropias Editorial S.L.

Redolar, D. (2012). El cerebro cambiante. Barcelona, España: Editorial UOC.

Rebolledo-Malpica, D. (2019). Enfermería según la perspectiva deconstructivista de Derrida. Revista de la Facultad de Medicina, 67 (1), 91-96.

Rubinstein, S. (1963). El ser y la conciencia: y el pensamiento y los caminos de su investigación. México: Editorial Grijalbo.

Sánchez, R., Martínez, V., González, M., Peiró, J., y Peiró, J. (2009). Un análisis transnivel de las relaciones de la calidad de servicio y la confirmación de expectativas con la satisfacción de los usuarios. Psicothema, 21(3), 421-426.

Taylor, S., y Bogdan, R. (2002). Introducción a métodos cualitativos de investigación. Barcelona, España: PAIDÓs.

Toro, I. (2006). Método y conocimiento: metodología de la investigación: investigación cualitativa/investigación cuantitativa. Bogotá: Universidad EAFIT.

Mercadotecnia. Disponible en http://www.imagenoptica.com.mx/pdf/revista33/lealtad.htm

Valdez, J. (20 de mayo, 2016). Competencias actitudinales. [Mensaje en un blog]. Recuperado de https://jennifervaldezzarate.wordpress.com/2016/05/20/competencias-actitudinales/ Vértice. (2010). Atención al cliente. Málaga, España: Editorial Vértice. 
Vigo, P. (2015). Actitud emprendedora y oportunidades de negocio: Capacidades necesarias para lograr el éxito empresarial. Pontevedra, España: Ideas propias Editorial S.L. 